\title{
Cognitive Sensor Networks Implementation in Medical Environmental Surveillance
}

\author{
Aleksandra Mateska \\ Faculty of Electrical Engineering and \\ Information Technologies \\ Rugjer Boshkovik bb \\ 1000 Skopje, R. Macedonia \\ +38972320591 \\ amateska@feit.ukim.edu.mk
}

\author{
Konstantin Chomu \\ Faculty of Electrical Engineering and \\ Information Technologies \\ Rugjer Boshkovik bb \\ 1000 Skopje, R. Macedonia \\ +38975685509 \\ konstantin.chomu@feit.ukim.edu.mk
}

\author{
Liljana Gavrilovska \\ Faculty of Electrical Engineering and \\ Information Technologies \\ Rugjer Boshkovik bb \\ 1000 Skopje, R. Macedonia \\ +38923099114 \\ liljana@feit.ukim.edu.mk
}

\begin{abstract}
Wireless communication services, including Wireless Sensor Networks (WSNs), emerge in healthcare environments with a variety of e-health applications collecting medical data and patient information. Possible WSN ambient surveillance in hospitals (which is of secondary importance) must consider the coexistence challenges with the wireless technologies that collect the medical data and minimize the impact towards the medical devices that operate in the same frequency band. To mitigate the interference problems WSNs may efficiently utilize the dynamic spectrum access techniques introduced by the cognitive radio (CR). This paper presents a proof-of-concept for CR enabled wireless sensor network deployed in medical surroundings. The proof-of-concept is performed on a Sun SPOT testbed platform. It demonstrates the WSNs ability to dynamically adjust the transmission parameters (i.e. frequency) thus minimizing the interference to the wireless systems of primary importance in the hospital environments.
\end{abstract}

\section{Categories and Subject Descriptors}

C.2.1 [Computer-Communication Networks]: Network Architecture and Design; Network Protocols

\section{General Terms}

Design, Performance

\section{Keywords}

Wireless sensor networks, healthcare, dynamic spectrum access, cognitive radio

\section{INTRODUCTION}

Among the possible enablers of various e-health solutions, the Wireless Sensor Networks (WSN) are one of the most promising technologies [1]. The WSNs facilitate low-cost and low-power wireless interconnection between different sensors providing diversity of applications, starting from patients monitoring up to disaster and failure recovery [2]. Introducing WSN applications in healthcare environments must take into account the coexistence with the wireless devices, which together with the telemedicine systems operate, in the unlicensed Industrial, Scientific and Medical (ISM) radio bands. Depending on the application requirements, the primary WSN objectives are either to transmit the data timely and simultaneously in time-critical applications (e.g. wireless ECG transfer representing application of primary importance), or to reliably send a small amount of data in various surveillance applications (e.g. ambient monitoring representing application of secondary importance) [3]. It is inevitably important to provide solutions that will allow critical data to be delivered timely and correctly. The Dynamic Spectrum Access (DSA) strategy enabled by the CR can be efficiently utilized to accomplish the different application requirements, as well as to mitigate the interference with the existing wireless technologies.

$\mathrm{CR}$, as defined by J. Mitola [3], is a context-aware intelligent radio that solves the spectrum scarcity problem through its capability of autonomous reconfiguration, learning from and adapting to the communication environment. CR exploits the underutilized spectrum resources in time or frequency and provides efficient dynamic spectrum access through the detection of spectrum opportunity and adaptation of the transmission parameters [4]. DSA can become very vital to accomplish the different network requirements in terms of capacity utilization, communication constraints and networks coexistence in the crowded wireless bands. For example, in the areas of healthcare and telemedicine, a high number of sensors that are used to monitor the vital signs of the patients should transmit their monitored results concurrently and timely. On the other hand the sensors used for environmental monitoring can allow data transfer delays. Moreover, hospitals are replete with sources of interference and many applications that require ultimate reliability to assure patient safety. DSA may be an efficient solution to minimize the interference between the wireless technologies and to offer optimized spectrum usage in the healthcare environments.

This paper presents solution for WSN based surveillance in hospital environment. The WSN is composed of sensor nodes that provide monitoring of the environmental parameters important in hospitals (i.e. ambient temperature, humidity, or level of $\mathrm{CO} 2$ ). These sensor nodes are IEEE 802.15.4 empowered and operate in the unlicensed $2.4 \mathrm{GHz}$ band. In such medical surroundings the telemetry systems that collect the health critical data are of primary importance. They consist of wireless devices operating in the same band, usually using IEEE 802.11 or IEEE 802.15.4 technology. The idea of the proposed concept is to enable the 
surveillance WSN network with DSA capabilities in order to minimize the interference it induces to the existing wireless systems of primary importance, including the telemetry systems. The cognition is performed through centralized dynamic spectrum access approach and dynamic adjustment of the sensor nodes transmitting frequency. A proof-of-concept is demonstrated on a Sun SPOT based WSN platform, where dynamic reconfigurability is verified and tested.

The rest of the paper is organized as follows. Section 2 presents the profile of the wireless hospital environments and Section 3 presents the main advantages and challenges of the $\mathrm{CR}$ implementation in WSNs. Section 4 depicts the WSN testbed setup and the measurements performed to evaluate the cognition capability, while Section 5 concludes the paper.

\section{WIRELESS SENSOR TECHNOLOGIES IN HOSPITAL ENVIRONMENTS}

Wireless Medical Telemetry Service (WMTS) is a wireless service defined in the United States by the Federal Communications Commission (FCC) for data transmission related to patient's health [5]. FCC defines communication in three specific bands, but these bands have not been internationally agreed and the proprietary devices cannot be marketed or used freely in countries other than the United States. To overcome this problem, many manufacturers provide devices that transmit data in the ISM bands such as $902-928 \mathrm{MHz}$ and, more typically, in $2.4 \mathrm{GHz}$ band. The telemetry equipments are of primary importance and usually operate in the $2.4 \mathrm{GHz}$ band using IEEE $802.11[6,7]$ or IEEE 802.15.4 radios [8, 9]. The hospital implemented nonmedical WSN applications are of secondary importance and should particularly take into account the coexistence problems with the related medical data collecting technologies [10,11].

Typical WSNs are built upon the PHY and MAC layer implementations defined by the IEEE 802.15.4 standard [12]. The standard is the basis for the ZigBee specification, which offers a complete networking solution by developing the upper layers above the IEEE 802.15.4 MAC [13]. Four of the IEEE 802.15.4 channels $(15,20,25$ and 26) fall between the often-used and non-overlapping 802.11 channels (1, 6 and 11) as shown in Figure 1. The remaining 12 of the overall 16 channels will be exposed to interference when IEEE 802.11 networks operate in the same surrounding. Additionally, various ZigBee networks may coexist in the same hospital environment, e.g. networks that provide telemedicine services as well as networks that offer monitoring and/or automation. The wireless networks can experience significant challenges in achieving reliable communication and appropriate techniques such as DSA can help mitigate the coexisting issues.

\section{COGNITIVE RADIO IMPLEMENTATION IN WIRELESS SENSOR NETWORKS}

WSNs may gain many competitive benefits from the opportunity to perform DSA. Typical WSNs have event-driven data communication activity, characterized by bursts of data traffic depending on the application characteristics. The common WSN architecture with dense deployment of the sensor nodes usually evokes data collisions and contention delay. In both cases, the CR enabled sensor nodes may opportunistically access multiple channels and alleviate these potential challenges. CR capable sensor nodes can change their operating parameters such as

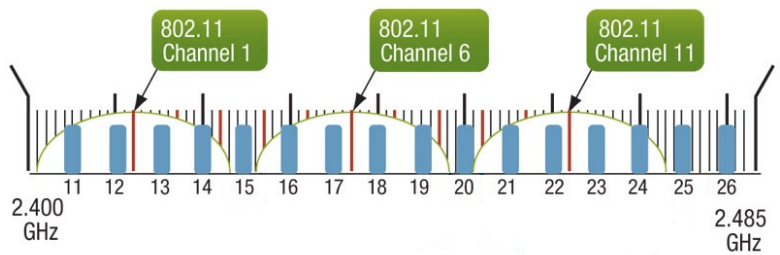

802.15.4 Channel

802.11 Channel $\quad 802.11$ Spectrum 0ccupancy (Typical)

Figure 1. Coexistence between IEEE 802.15.4 and IEEE 802.11 technologies

transmission power and, hence, reduce the power consumption used for data transmission and reception. Dynamic spectrum management may also significantly contribute to the efficient coexistence of spatially overlapping sensor networks in terms of communication and resources utilization [14].

CR implementation in WSN requires dynamic spectrum access framework to perform the main CR functionalities, i.e. spectrum sensing, spectrum decision and spectrum handoff. Spectrum sensing is fundamental CR feature that needs to be implemented in the WSN nodes since they must gather spectrum occupancy information and make appropriate decisions about the available spectrum. Among the various spectrum sensing techniques (e.g. match filter, energy detection or feature detection), energy detection is preferred in WSNs due to the nodes' processing and power constraints. The sensor nodes measure the receiver energy on a specific spectrum band (channel) for a certain period of time and decide upon the channel availability comparing the received energy with a predefined threshold.

When the spectrum sensing is performed, the sensor nodes analyze the sensing data and make a decision about transmission parameters (channel, transmission power and/or modulation). Decision making algorithms for WSNs must consider the limitations of the sensor nodes hardware as well as the ad-hoc nodes organization, coordinating the spectrum decisions in centralized or distributed approaches. With the centralized coordination methods, the decision making is performed by the sink node after receiving the spectrum occupancy measurements from the distributed sensors. The distributed approaches are also suitable for ad-hoc WSNs, where the nodes share the spectrum sensing information with their neighbor nodes and locally decide about the spectrum occupancy. The centralized methods usually lead towards optimal spectrum utilization since the sink node has global network information, but additional control messages may influence the power consumption as well as the overall data communication. Upon deciding on the available channels, the nodes perform spectrum handoff or spectrum mobility. Depending on the hardware performances, the handoff may cause long delays or buffer overflows reflecting on the application performances.

This paper aims to demonstrate that WSNs can provide reliable and non-intrusive surveillance in medical premises when enabled with CR capability.

\section{SCENARIO \& TESTBED SETUP}

This section presents the testbed setup based on Sun SPOT platform that demonstrates the DSA capability and flexibility of the surveillance equipment. The aim is to demonstrate the WSN ability to change the RF transmission properties (i.e. transmission frequency), depending on the spectrum occupancy, in order to 
avoid the channels/frequencies used by the other wireless technologies that operate in the same unlicensed band. Sun SPOT surveillance data is treated as secondary user (SU) ZigBee transmission in the scenario where transmissions from medical applications (ether using IEEE 802.11 or IEEE 802.15.4 technology) are of primary importance.

Sun SPOTs perform several enabling DSA capabilities:

- $\quad$ Spectrum sensing

- $\quad$ Spectrum decision

- $\quad$ Spectrum handoff

These features are implemented in Sun SPOT and host applications. The Sun SPOTs perform spectrum sensing and handoff, while the decision making is performed with a centralized approach by the host application.

\subsection{Scenario description}

The aim of the WSN network implementation in hospitals is to provide surveillance and real-time monitoring of the environmental parameters important for the medical surroundings. The scenario architecture is depicted in Figure 2a. The WSN nodes, i.e. Sun SPOTs $[15,16]$, are equipped with sensors that perform the sensing of the physical phenomena (such as temperature, humidity, $\mathrm{CO} 2$ ), processors that perform the in-node data processing and transceivers that send the data wirelessly to the sink node (Figure 2b). The sink, also called Base Station (BS), is connected to a PC and is driven by the host application.

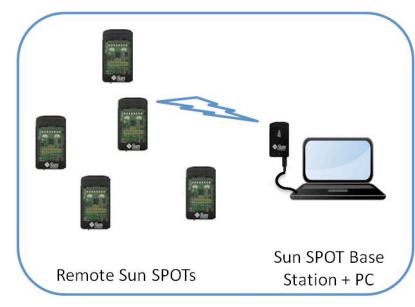

a. Testbed architecture

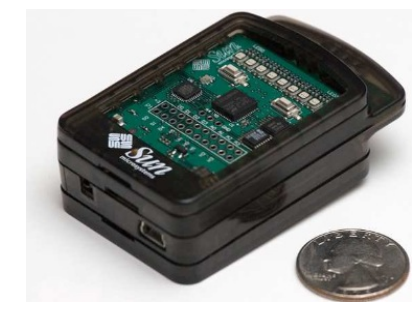

b. Sun SPOT
Figure 2. Sun SPOT testbed architecture

The telemedicine applications are of primary importance in hospitals since they have firm and critical demands regarding the data communication. The WSN application used for surveillance is treated as a second-degree network, as it may allow certain amount of data delay and packet losses without degrading the overall services quality. In order to avoid the interference that the surveillance WSN network causes to existing wireless systems, regardless the technology they use, the sensor nodes are enabled with DSA capability and may choose a transmission channel, which is not used by the other technologies.

The DSA is performed using energy detection for spectrum

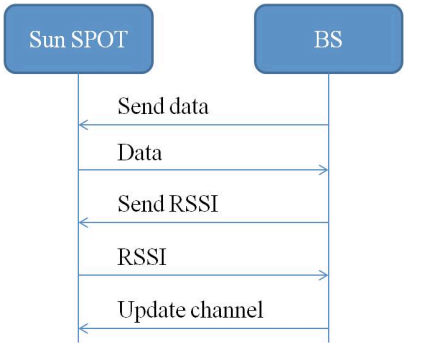

Figure 3. Message sequence chart sensing, centralized decision making and spectrum handoff driven by the exchange of control messages between the WSN nodes (Figure 3). Two types of applications perform the cognitive channel selection, i.e. on-spot and host applications. Sun SPOTs (through the on-spot application) send data and control messages - Data and RSSI, and the BS (through the host application) broadcasts three types of control messages - Send data, Send RSSI and Update channel.

\subsection{Testbed setup}

The laboratory tesbed (Figure 4) is set up according the testbed architecture presented in Figure 2a. The Sun SPOTs measure the spectrum occupancy and the centralized decision making device (BS) drives the spectrum handoff aiming to switch to unoccupied channel for surveillance data transmission (SU data).

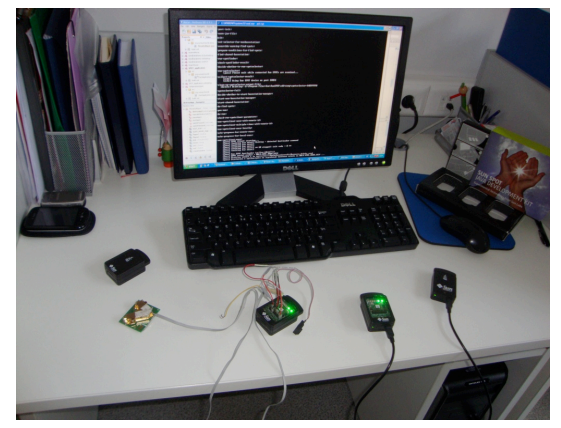

Figure 4. Scenario setup

The Sun SPOTs send Data messages with the environmental parameters when receiving the initial Send data message from the BS. After receiving Send RSSI message, they measure the RSSI level on each of the 16 channels with adjusted spectrum measurement approach presented in [16, 17]. These RSSI measurements are used by the decision making device (BS) to decide upon available frequencies for further transmissions. Table 1 presents the testbed parameters

TABLE 1. Testbed parameters

\begin{tabular}{ll}
\hline \hline Number of Sun SPOTs & 5 \\
\hline Data messages interval & $10 \mathrm{~s}$ \\
\hline Send RSSI message interval & $5 \mathrm{~s}$ \\
\hline Operating bandwidth & $2405 \mathrm{MHz}$ to $2480 \mathrm{MHz}$ \\
\hline Number of channels & 16 \\
\hline Channel width & $2 \mathrm{MHz}$ \\
\hline Dwell time & $10 \mathrm{~ms}$ \\
\hline Transmission power & $-3 \mathrm{dBm}$ \\
\hline \hline
\end{tabular}

Initially, the surveillance information is send via unoccupied channel, i.e. the channel with minimum activity. If medical data transmission occurs in that particular channel, the transmission of the surveillance data is switched to the next free channel (channel with minimum averaged RSSI level). BS sends Update channel message to announce the channel update. After receiving the Update channel message, the nodes perform the spectrum handoff and proceed to communicate on the new data channel thus avoiding interference to other technologies, and to any other 


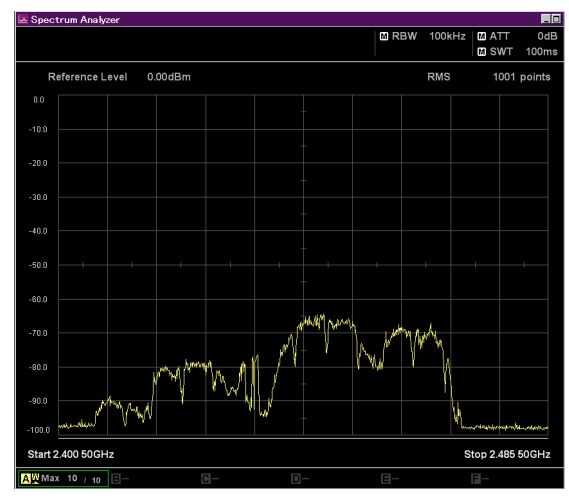

a. Initial spectrum occupancy

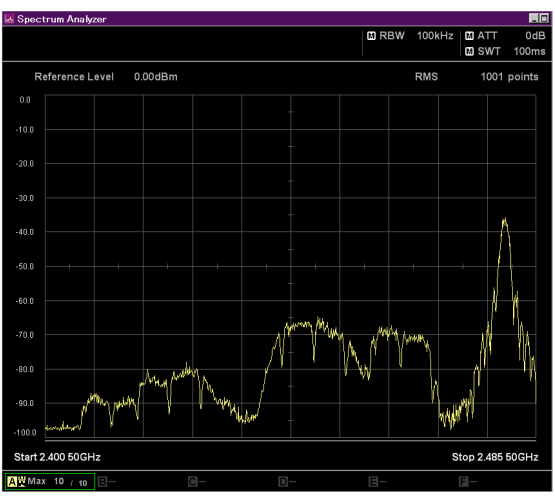

b. Spectrum occupancy before spectrum handoff

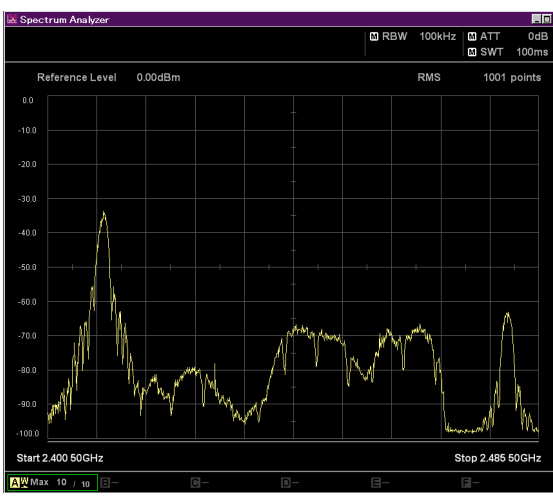

c. Spectrum occupancy after spectrum handoff

Figure 5. 2.4GHz spectrum occupancy measurements

transmissions (including based on the same technology) that might transmit any medical data.

\subsection{Performance analyses}

Spectrum measurements are performed with Spectrum analyzer in the Sun SPOTs vicinity. The measurement setup demonstrates surveillance data spectrum handoff initiated by medical ZigBee data transmissions from collocated equipment. Figures 5-7 demonstrate the testbed behavior.

The initial spectrum measurements demonstrate the permanent IEEE 802.11 activity in the $2400-2485 \mathrm{MHz}$ ISM band (Figure $5 a)$. The Sun SPOTs ZigBee surveillance activity on Channel 26, before the spectrum handoff, is presented in Figure $5 \mathrm{~b}$. Figure $5 \mathrm{c}$ depicts the ZigBee medical transmission on Channel 26 that causes the spectrum mobility, and the surveillance data transmission on Channel 12 after the spectrum handoff.

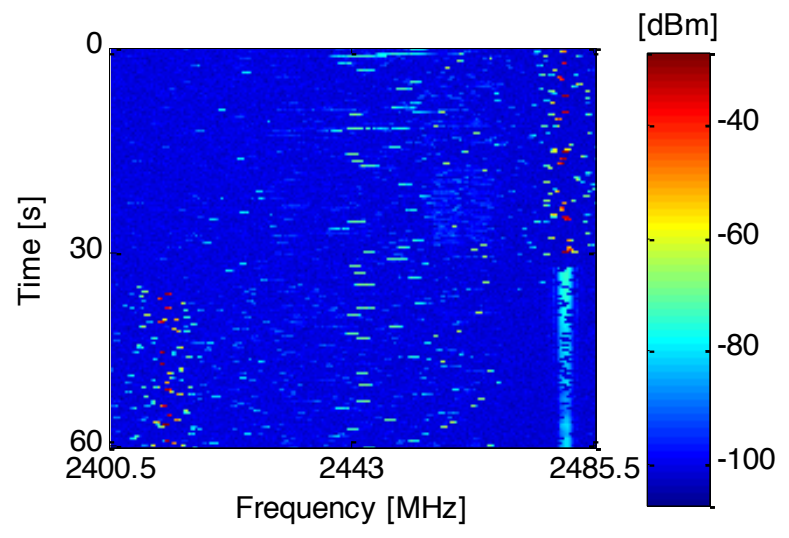

Figure 6. Waterfall plot of the $2.4 \mathrm{GHz}$ spectrum activity

Figure 6 presents the waterfall plot of the spectrum activity (frequency vs. time) and depicts the Sun SPOTs channel switching and the continuum of data transmission (at $t=30 \mathrm{~s}$ ). Analog to Figure 5, the waterfall demonstrates the Sun SPOTs ZigBee activity during the spectrum mobility, i.e. before and after the change of the ZigBee transmission frequency. Figure 7 presents the received signal strength on the two data channels (26 and 12), without the Sun SPOT data transmissions. The figure depicts the moment when the activity on Channel 26 that has resulted in higher RSSI level (at $\mathrm{t}=30 \mathrm{~s}$ ) initiates the Sun SPOTs switch to Channel 12.

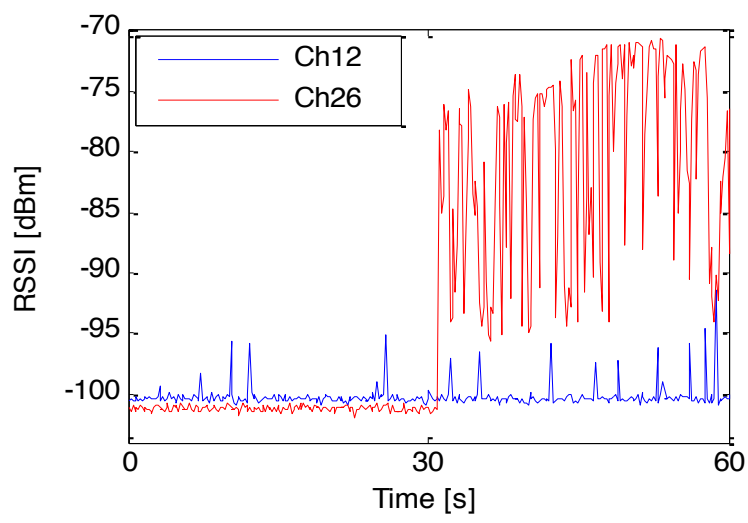

Figure 7. RSSI level on Channels 12 and 26 without Sun SPOTs activity

The presented approach demonstrates centralized cognitive decision making procedure, which drives the WSN surveillance network to minimize the interference to the coexistent wireless systems in hospital environments. The presented proof-of-concept efficiently performs the cognitive dynamic spectrum access strategy by dynamically choosing minimally utilized spectrum bands. The measurements verify the WSN capability and the Sun SPOTs performances towards cognitive sensor networks implementation in medical environmental surveillance.

\section{CONCLUSION}

This paper presents the DSA capability of WSNs deployed in medical surroundings, which are demonstrated on a Sun SPOT based testbed. Sun SPOT nodes perform spectrum sensing, spectrum decision and channels handoff with centralized DSA approach. The dynamic adjustment of the sensor nodes transmitting frequency minimizes the interference that the WSN network imposes on the other wireless coexistent systems. Using the proposed DSA approach, the surveillance network minimizes the impact towards any wireless network that operates in the same surrounding; also enabling reliable data transfer for the applications of primary importance in hospitals (e.g., patients' telemetry services). 
The expansion of the presented concept into real hospital surroundings, where the WSN nodes will be spatially distributed in larger area, may require certain adaptations. The decision making algorithms regarding the spectrum occupancy should take into account the network spatial distribution and utilize different

\section{REFERENCES}

[1] Stankovic S. 2010. Medical Applications Of Wireless Sensor Networks:Who-Did-What. Application and Multidisciplinary Aspects of Wireless Sensor Networks: Concepts, Integration and Case Studies. Eds. L. Gavrilovska; S. Krco; V. Milutinovic; I. Stojmenovic; R. Trobec. Springer (2010), 188-202.

[2] Akyildiz, I. F., and Vuran M.C. 2010. Wireless Sensor Networks. Wiley

[3] Mitola J. 1999. Cognitive radio for flexible mobile multimedia communications. Mobile Multimedia Commun. Proc. -MoMuC '99, (Nov. 1999). 3-10.

[4] Zhao Q. and Sadler B. M. 2002. A Survey of Dynamic Spectrum Access. IEEE Signal Processing Magazine. 24,3 (May 2007), 79-89. DOI= 10.1109/MSP.2007.361604

[5] Wireless Medical Telemetry Service, Federal Communications Commission. Available at: http://www.fcc.gov/encyclopedia/wireless-medicaltelemetry-service-wmts

[6] Gibson M. 2005. Healthcare Facility Measurements A Case Study: Radio Frequency Site Survey, Spectrum Analysis, and Measurements in the Hospital. White Paper, Comsearch, Andrew Corporation (2005).

[7] Anritsu. 2011. Radio Frequency Interference in Hospitals. Application Note. Rev. A Printed in United States 2011-07, C2011 Anritsu Company.

[8] ZigBee Wireless sensor Applications for Health, Wellness and Fitness, ZigBee Alliance, 2009. Available at: https://docs.zigbee.org/zigbee-docs/den/09-4962.pdf model-based or learning techniques. Also, transmit power adaptation may be performed, thus increasing the overall utilization and maximizing the power efficiency.

[9] Tam V. N. 2008. Medical Applications of Wireless Networks. Available at: http://www.cse.wustl.edu/ jain/cse57408/ftp/medical/index.html

[10] Zhang X., and Shin K. G. 2011. Enabling Coexistence of Heterogeneous Wireless Systems: Case for ZigBee and WiFi. ACM Trans. Program. MobiHoc'11, 6, (May 2011). DOI= http://dl.acm.org/citation.cfm?id=2107510

[11] Mardini W., Khamayseh Y., Jaradatand R., and Hijjawi R. 2012. Interference Problem between ZigBee and WiFi. In IPCSIT. 30 (2012).

[12] IEEE 802.15.4 Standard on Wireless MAC and PHY Specifications for Low-Rate WPANs. Available at: http://www.ieee802.org/15/pub/TG4.html

[13] Baronti P., Prashant P., Chook V., Chessa S., Gotta A., and Fun Hu Y. 2007. Wireless Sensor Networks: A Survey on the State of the Art and the 802.15.4 and ZigBee Standards. Computer Communications. 30, (2007), 1655-1695.

[14] Akan O. B., Karli O. B., and Ergul O. 2009. Cognitive radio sensor networks. IEEE Network. 23, 4 (July-August 2009), $34-40$.

[15] SunTM Small Programmable Object Technology (Sun SPOT) Developer's Guide, Sun Microsystems, 7 May 2009.

[16] Chomu K., Mateska A. and Gavrilovska L. 2011. Sun SPOT based WSN Testbed. ETAI (2011).

[17] Denkovski D., Rakovic V., Pavloski M., Chomu K., Atanasovski V. and Gavrilovska L. 2012. Integration of Heterogeneous Spectrum Sensing Devices towards Accurate REM Construction. IEEE Wireless Communications and Networking Conference - WCNC'12, (April 2012). 